\title{
A Fault Diagnosis Method for Out-of-Round Faults of Metro Vehicle Wheels with Strong Noise
}

\author{
Haifeng Huang $\mathbb{D}^{1},{ }^{1}$ Heli Wang $\mathbb{D},^{2}$ Weijiu Zhang, ${ }^{3}$ and Weijie Gu $\mathbb{D}^{1}$ \\ ${ }^{1}$ School of Mechanical Engineering, Southwest Jiaotong University, Chengdu 610031, China \\ ${ }^{2}$ Patent Examination Cooperation Sichuan Center of the Patent Office, China National Intellectual Property Administration, \\ Chengdu 610213, China \\ ${ }^{3}$ Wuhan Metro Operation Co., Ltd., Qiaokou District, Wuhan 430000, China
}

Correspondence should be addressed to Haifeng Huang; hhfeng@swjtu.edu.cn

Received 13 June 2021; Revised 24 August 2021; Accepted 26 August 2021; Published 4 October 2021

Academic Editor: Chengwei Fei

Copyright (c) 2021 Haifeng Huang et al. This is an open access article distributed under the Creative Commons Attribution License, which permits unrestricted use, distribution, and reproduction in any medium, provided the original work is properly cited.

\begin{abstract}
Detection of out-of round (OOR) faults of metro vehicle wheels is very important to improve stationarity and stability in metro vehicles and avoid accidents caused by OOR faults. Diagnosis of OOR faults demands extracting useful information accurately from mass of vibration signals with poor signal-to-noise ratio (SNR) of metro vehicle wheels for complex running condition. In this paper, we proposed a diagnosis method on OOR faults of metro vehicle wheels combined with variational mode decomposition (VMD), kernel principal component analysis (KPCA), and deep belief network (DBN) to diagnose the OOR faults of metro wheels. Vibration signals of China metro vehicle wheels collected while the metro vehicle is running are used to train the diagnosis model and adjust parameters of DBN and KPCA based on testing accuracy. The different dimensions of KPCA, epoch number, and node number of DBN are compared, and the better parameters of diagnosis model based on vibration signals are concluded in this paper. The generalization of the diagnosis model is checked nine times by testing the calculation of each group of parameters and using an error declining process. The mean accuracy of diagnosis model proposed in this paper is 0.9136 , and the diagnosis model presented in this paper is very significant to detect OOR faults online.
\end{abstract}

\section{Introduction}

Metro vehicles are one of urban transportation modes in most large and medium-sized cities for metro vehicles' advantages of safety, fast speed, and comfort. However, wear and fatigue damages of wheels will be unavoidable for the running of metro vehicles and thus lead to out-of-round (OOR) faults of metro vehicle wheels. OOR faults of wheels impact rails and enhance vibration of metro vehicles, especially vertical vibration. The increasing vertical vibration and impact can also cause critical damages on contact surface of wheel-rail, which will also shorten a service life and even lead to damages of many key components of the vehicle system, such as axle box bearing and braking system. Those damages and shortened life of key components cannot ensure safe running of metric vehicles [1], and increased vertical vibration and impact between rail and wheel may even cause derail accidents. Nowadays, people pay more attention to detection, collecting fault signals, diagnosis, and failure causes of OOR faults [2-6]. However, it is hard to separate fault information from complex vibration signals collected by vibration sensors during metro vehicles running.

Song et al. introduced some relationships between outof-round faults (OORF) and vertical dynamic performance, and the secondary suspension of the vehicles has no damp function caused by OORF [7]. Lan et al. [1] focused on the effect of OOR faults for wear of wheels under normal braking condition. The wheels would slid, and the braking torque dominate wear in braking condition. They also took wheel unloading ratio as an indicator for the determination of risk to derailment when the wheel contains OOR defect. Tao [8] investigated OOR faults of electric locomotive wheels through extensive measurement conducted at field sites. The 
measurement results indicate that two types of freight traffic locomotives suffer serious polygonal wear problems with center wavelengths ranging from 160 to $315 \mathrm{~mm}$ and the dominating wavelength is $200 \mathrm{~mm}$.

Fu et al. identified the types of wheel faults in urban rail trains based on improved ensemble empirical mode decomposition (EEMD) and Hilbert transform [9]. Zhang et al. proposed a method of the online fault diagnosis of the train wheels by characteristic spectrum analysis with noisy background [10]. They all focused on extracting fault characteristic under noisy background of trains.

Many excellent fault diagnosis methods are proposed although those methods are not used to diagnose OOR of train wheels. G. Adam et al. diagnosed ventilation faults of angle grinder by means of infrared (IR) thermography and IR image processing [11]. AlShorman et al. reviewed condition monitoring and fault diagnosis based on sound and acoustic emission for four types of faults: bearings, rotor, stator, and compound [12]. Mass Laplacian discriminant analysis was used to diagnose faults of gear and had a higher diagnostic accuracy [13]. Yang et al. proposed a novel multilayer domain adaptation method to diagnose the compound fault and single fault of multiple sizes simultaneously [14].

All above, vibration signal, acoustics signal, and thermal imaging are used as input signals of fault diagnosis. People will choose one or more types of signals as input according to the equipment structures, sensor locations, working condition, and types of noise.

Monitoring and diagnosing out-of-round faults of wheels accurately are very important for metro vehicle's safe running and reducing maintenance time and costs. In order to diagnose accurately OOR faults of metro vehicles online, we may build a system which includes noise reduction, extraction of components of faults, improving diagnosis accuracy, and online speed.

Deep learning is often used to predict passenger flow and safety prediction of rail transit system [15-17], and we try to combine variational mode decomposition (VMD), kernel principal component analysis (KPCA), and deep belief network (DBN) to detect OOR faults of metro wheels in this paper.

In this paper, we proposed a diagnosis method on OOR faults of metro vehicle wheels combined variational mode decomposition (VMD), kernel principal component analysis (KPCA), and deep belief network (DBN) and match parameters of the diagnosis model to diagnose the OOR faults of metro wheels.

\section{Vibration Signals of Metro Vehicle's Wheels}

OOR faults of wheels may mainly cause vertical vibration of wheels, but those faults are not the only factors of vertical vibration. Irregularity state of railway and faults of rolling bearings of axle box can also cause vertical vibration when the vibration sensors are located on the axle box. Irregularity state of railway and OOR faults of wheel are shown in Figures 1 and 2. Reasonable locations of sensors, effective signal collection, and accurately separating vertical vibration

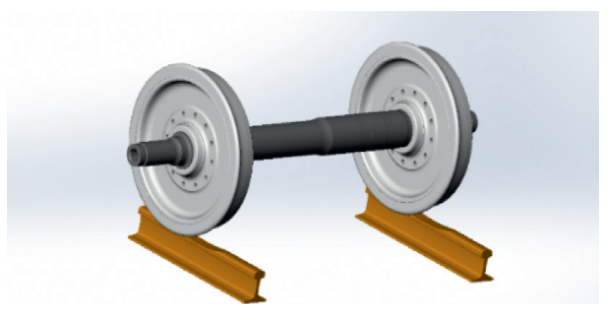

Figure 1: Irregularity state of railway.

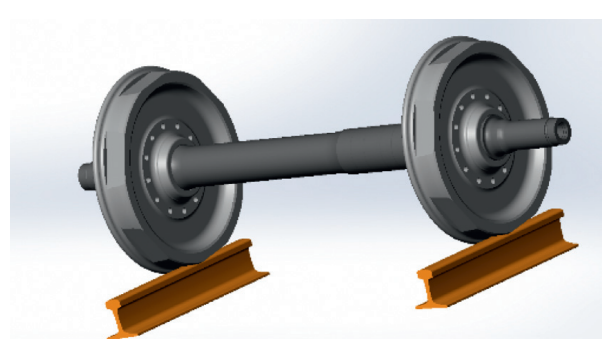

FIGURE 2: OOR faults of wheels.

of OOR faults are very important for diagnosing OOR faults of metro vehicle wheels.

2.1. Collection of Vibration Signals of Metro Vehicle's Wheels. Reasonable locations of sensors can help to collect effective vibration signals, which will contain state information of OOR faults as much as possible. That means vibration sensors should be placed near to the vibration sources. Four vibration sensors are located on axle boxes of four wheels, respectively, as shown in Figure 3.

The four vibration sensors are mainly used to collect vertical vibration signals for OOR faults of wheels. Vertical vibration of wheels may include vibration come from other vibration sources, such as irregularity state of railway and vibration of metro vehicle's body. The vibration sensors will collect all the vertical vibration signals caused by all factors, which are mixed and collected by vibration sensors and include lots of noise. Furthermore, driving components including gear box and motor will also produce vibration while metro vehicle is running, and the final collected vibration signals are mixed with vibration of motor and gear box. As shown in Figure 1, vibration signals may show difference in amplitude and frequency by sensors 1 and 2 when they are located in two axle boxes, because sensor 1 is near to the gear box and motor, while sensor 2 is far from them.

When the metro vehicles are running with good state, collected vibration signals by vibration sensors will include vertical vibration caused by lots of vibration sources, such as irregularity state of railway and vibration of metro vehicle's body, and not only that caused by OOR faults of wheels. In practice, vibration signals caused by different vibration sources usually overlap and interact with each other, and separating vibration signals of OOR faults from measured vibration signals collected by sensors is the preprocessing work for diagnosing OOR faults of metro vehicle' wheels. 


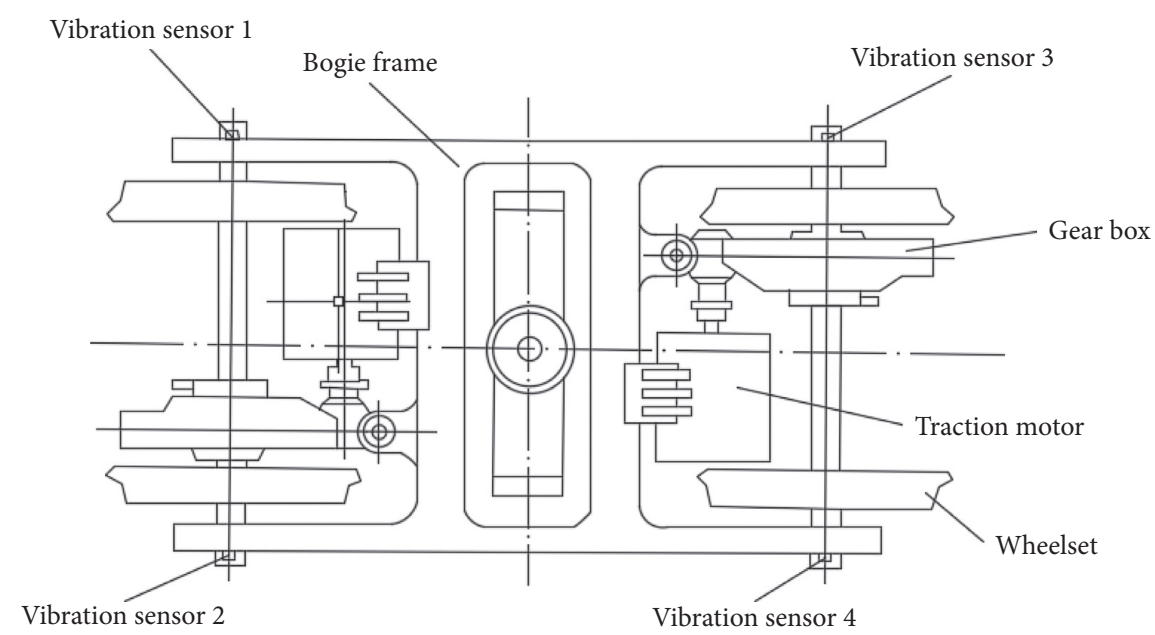

Figure 3: Locations of vibration sensors.

In this paper, a separating principal component of OOR faults and denoise method is presented to get rid of interfering vibration of gears, motors, and vehicle bodies. We propose a method of KPCA-DBN to diagnose OOR faults rapidly and accurately without artificial feature extraction and selection for effective information of raw data being kept by KPCA $[16,17]$. We choose matching parameters of DBN and dimension of KPCA adapting vibration signals of metro vehicle's wheels to build a diagnosis method of OOR faults.

2.2. Vibration Signals of Metro Vehicle's Wheels. We choose three groups of vertical vibration signals, which are three states of normal, incipient OOR faults, and serious OOR faults of metro vehicle's wheels. Signal-to-noise ratio (SNR) is below five when wheels are with incipient or serious OOR faults (Table 1). Ten datasets of each group of vibration signals are chosen to test the method of diagnosis and denoise proposed in this paper. The waves of vibration signals collected by sensors in normal, incipient, and fault states are shown in Figures 4-6, in which there are four waves for each state without denosie and extracting principal component. Spectra display of vibration signals are shown in Figures 7-9, which are spectra display when wheels are normal, incipient fault, and fault state, respectively.

2.3. Vibration Signals Processing. The SNRs of vibration signals of incipient faults and faults states collected by sensors are no more than four, which are all low SNR signals with strong noise. That means fault information may submerge in strong noise, and we will enhance SNRs of vibration signals by choosing wavelet threshold denoising function and setting an optimal number of wavelet decomposition layers to get rid of strong noises. Then, we evaluate the effect of wavelet denoise by comparing SNR and root mean square error between the original and denoising signals.

\section{Fault Diagnosis Model Based on DBN}

3.1. Structure and Pretraining of DBN. DBN is often used for predicting and diagnosis [18-20], which is composed of restricted Boltzmann machines (RBM), and the number of layers of DBN will increase with increasing the number of RBMs. Usually, DBN has multiple hidden layer structure with multiple RBMs, and the first RBM is the visible layer of DBN and the output layer of first RBM is the input layer of the second RBM. By this way, two adjacent RBMs are connected and the output layer of last RBM is the output layer of DBN (Figure 10. DBN can extract features of the data step by step and can realize deep learning $[21,22]$.

Greedy algorithm [23] and backpropagation algorithm (BP) are combined to train DBN so that the optimal initial weight can be found and get deep information of data. BP is adopted to adjust the initial weight for global optimization.

We will select proper number of layers, numbers of nodes, and step size when DBN is used to diagnose faults of wheels. Otherwise, underfitting or overfitting may appear during learning for being not the best initial value, data distribution, and other factors.

We try to match characters of DBN and vibration signals of wheels with different wheel states by the following ways.

(1) Greedy algorithm and backpropagation algorithm (BP) are combined to train DBN, and BP is adopted to adjust weights for global optimization.

(2) The structure of DBN is simplified into two RBMs in diagnosing fault of OOR based on distributions of vibration signals to escape overfitting.

(3) Upset all data so that training data and testing data have the same distributions to escape underfitting.

(4) Collected vibration signals by sensors may include much more noises when metro vehicles are running, and the noises may affect mapping between input and output of the DBN model. In this paper, wavelet and VMD are combined to get rid of noises. 
TABLE 1: SNRs of vibration signals without denoise and extracting principle principal component.

\begin{tabular}{lcc}
\hline Vibration signals of normal state & Vibration signals of incipient faults state & Vibration signals of faults state \\
\hline 12.67 & 1.85 & 3.96 \\
\hline
\end{tabular}
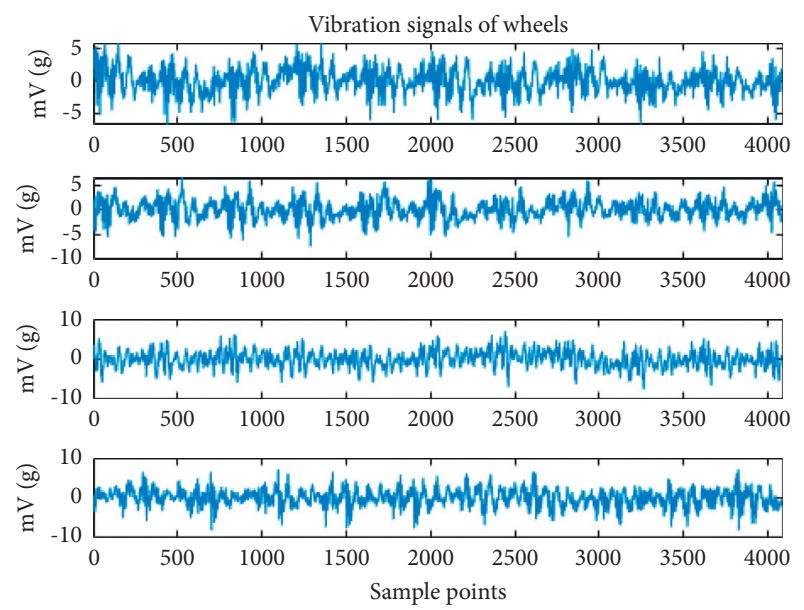

FIGURE 4: Vibration waves of normal wheels.

(5) Try to select fit training step and training times to avoid overfitting and underfitting.

In addition, we select appropriate number of KPCA to increase diagnosis accuracy of OOR and combine wavelet and regularization method to increase generalization of diagnosis model.

3.2. Denoise Processing by Wavelet and VMD. Frequency range of wheels with OOR can be estimated by wheel diameter and running speed of metro vehicle. Wheel diameter is $840 \mathrm{~mm}$. We collect vibration signals when wheel speeds are from $92 \mathrm{r} / \mathrm{min}$ to $592 \mathrm{r} / \mathrm{min}$, and then, we can calculate speed of metro vehicles to be from $14.56 \mathrm{~km} / \mathrm{h}$ to $93.69 \mathrm{~km} / \mathrm{h}$ as follows:

$$
v=\frac{60 \pi d n}{1000}(\mathrm{~km} / \mathrm{h}) .
$$

Frequencies of vibration signals caused by OOR faults can be expressed by

$$
f=\frac{N n}{60}(\mathrm{~Hz}),
$$

where $N$ is an integer which is affected by wheels' states and if there are much more OOR faults, $N$ will be larger than that there are less OOR faults in wheels. $n$ is the rotation speed of wheels. When $N$ equals one, the main frequency range caused by OOR faults may be between $1.53 \mathrm{~Hz}$ and $9.87 \mathrm{~Hz}$ for different running speeds of metro vehicles.

We adapt the variational mode decomposition (VMD) to decompose vibration signals into five intrinsic mode functions (IMFs) and reduce noise signals for IMF1 by wavelet shown in Figure 11. The envelope diagram in
Figure 11(b) shows more obvious frequency characteristic than that of Figure 11(a), which is processed by wavelet.

3.3. OOR Fault Diagnosis Model. Vibration signals of metro vehicle wheels collected by sensors are reduced noises by denoise process in Figure 12 which are input data for building OOR fault diagnosis model. $70 \%$ of the data is used to train DBN after extracting the main components by KPCA. KPCA dimension number is reduced to be 22 for reducing calculation and overlap. Then, we tried to adjust the parameters of DBN to match the data of vibration data of metro vehicles and get more accurate diagnosis accuracy on OOR faults of wheel sets. Layer number, node number, literation number, and epoch number should be adjusted to meet the data of metro vehicles, when we get the best parameters of DBN and we can get the fault diagnosis model of OOR fault of metro vehicle wheels. The model can be used to distinguish whether there are OOR faults on wheels or not.

\section{Testing Diagnosis Model by Metro Vehicle Vibration Data}

We tested the diagnosis model combined by wavelet, VMD, KPCA, and DBN methods using three groups of metro vehicle vibration data, which include normal, incipient, and fault wheels' vibration signals collected by vibration sensors, and each state data group includes 10 sets of vibration signals. There are 1024 points in each set of vibration signal. First, we mix all vibration signals and divide them into training datasets and testing datasets again. By dividing the datasets again and all the data is mixed with random turn, overfitting may be declined and diagnosis accuracy can be increased.

All the vibration signals are reduced noise signals by wavelet and VMD, and then, signals are declined dimensions by KPCA. All the data are divided into 40 sets after being mixed. Second, we choose two RMBs of DBN with 30 nodes of the first layer and 20 nodes of the second layer. The learning rate is 0.001 , and the iterations are 1000 . Training sets are input into diagnosis model of OOR faults, and testing sets are input into the trained model to calculate the diagnosis accuracy, which is 0.8611 . Errors of testing test 1 nine times are declined with iterations (Figure 13. Those of test 2 are shown in Figure 14, those of test 3 are shown in Figure 15, those of test 4 are shown in Figure 16, those of test 4 are shown in Figure 17 and those of test 5 are shown in Figure 18.

Though the same data and the same DBN parameters are taken in the same test, the error decline process of each testing is different for DBN and random initial weight values may be assigned to each time test calculation.

First, choose the dimensions of KPCA to be 40 and the node number of first layer to be 50 and that of second layer to be 30 ; 


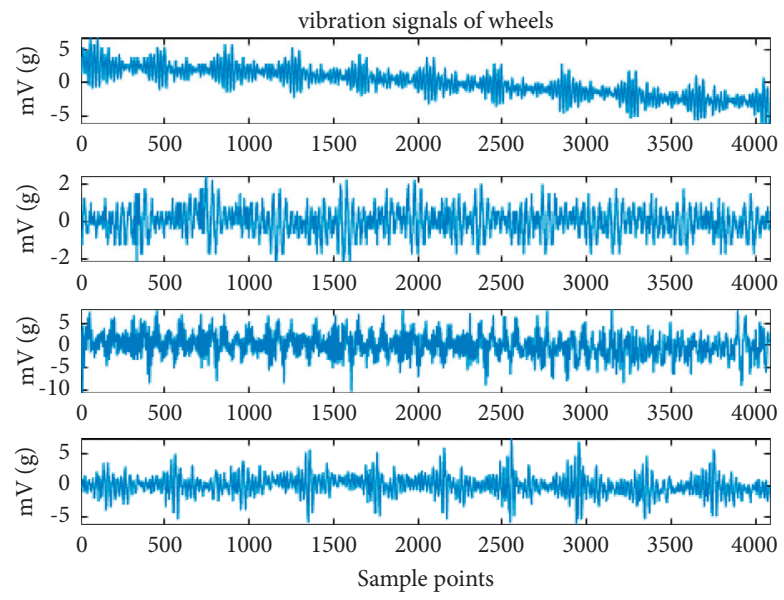

FigURE 5: Vibration waves of incipient faults wheels.
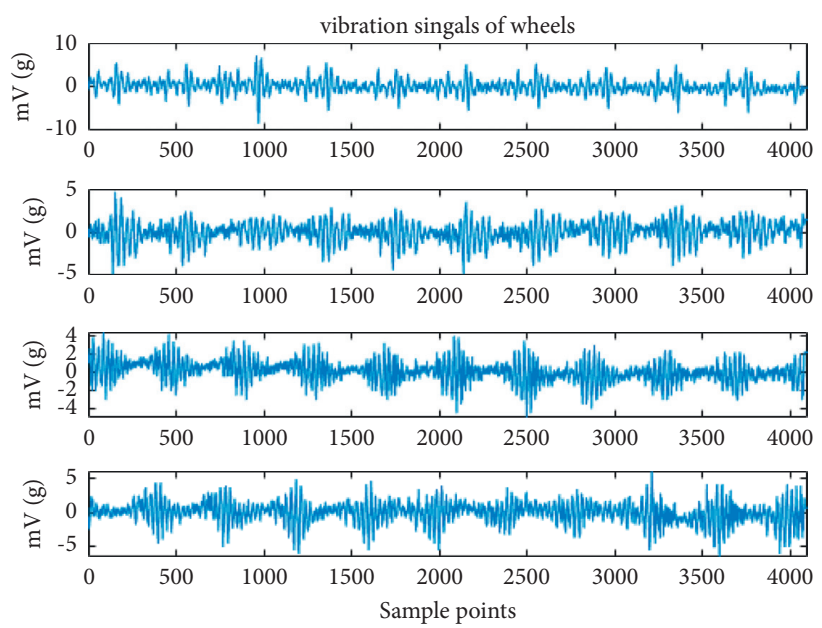

FIGURE 6: Vibration waves of faults.

using these parameters, the testing accuracy of diagnosis is 0.80565 . Second, change the dimensions of KPCA to be 20 and the node number of first layer to be 30 and that of second layer to be 20; using these parameters, the testing accuracy of diagnosis is 0.8611 . Third, change the dimensions of KPCA to be 25 and the node number of the first layer to be 20 and that of second layer to be 20 . Then, the testing accuracy of diagnosis is 0.8889 . We continue to change the dimensions of KPCA to be 22 and the node number of the first layer to be 30 and that of second layer to be 20 . By those parameters, the testing accuracy of diagnosis is increased into 0.9444. By further changing the dimensions of KPCA to be 25 and other parameters not changed, the diagnosis accuracy is 0.9722 (Table 2 . In the sixth test, the node number of the second layer is enhanced to be 30 and the other parameters are the same as test 5 .

Each test is calculated nine times to check the generalization of the diagnosis method proposed in this paper, and the average accuracy of test 6 is 0.9136 , which is the highest average accuracy in all tests. The results of the calculation show that the parameters of test 6 are the best optimal parameters of all the tests.

Test 6 may have the best generalization for ORR diagnosis of metro vehicle. The number of iterations is enhanced to be 1500 , and the other parameters are as the same as test 6 , and the average accuracy almost equals 1000 literation times. That means that 1000 literation times are enough for the vibration signals of metro vehicles taken in this paper.

In every test, the data have been calculated 1000 times in one epoch, and many epochs may be taken in training DBN model for each time of the calculation. We calculated each test nine times (Table 3 . We chose 60, 45, 20 epochs for test 6 to find how test accuracy is going with different epoch times (Figure 19. When epoch time is 20, the test accuracy is more stable for different calculation times and 20 epochs may be the best epoch times among 60,45 , and 20 . When the epoch time is 45 and 60 , there are all the calculation accuracies below $80.00 \%$. This means that when epochs are chosen to be 45 and 60 , overlap may occur and calculation accuracy declines with the enhancing of epochs. Comparing with calculation results, we chose the epoch times to be 22 in this paper.

We chose the parameters of test 6 with 22 epochs and two RBMS of 30 and 30 for DBN, which, combined with KPCA method, are taken to be the OOR fault diagnosis model of metro vehicles in this paper. 

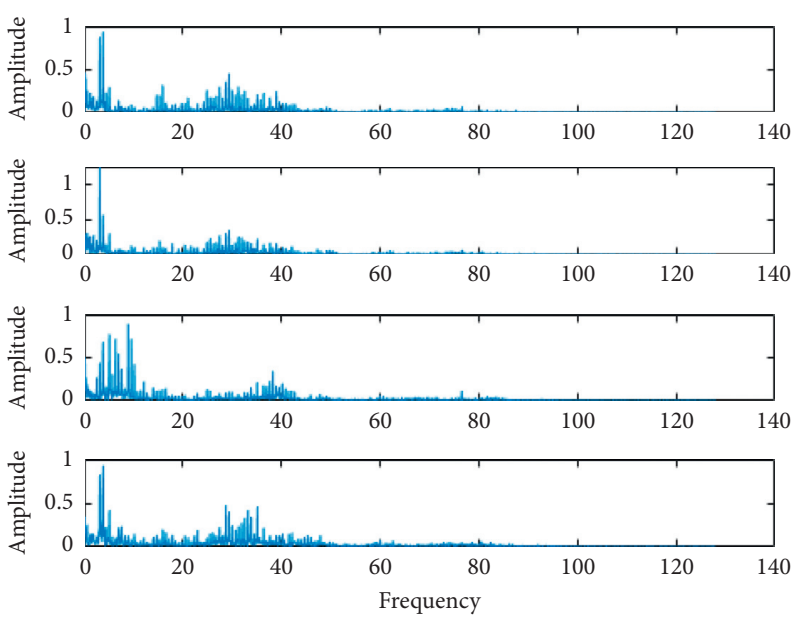

FIGURE 7: Spectra display of normal signals.
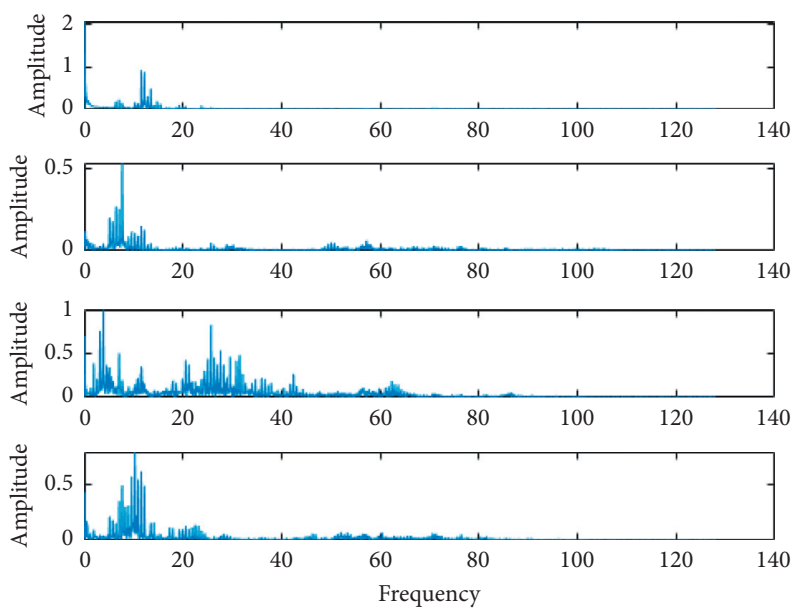

Figure 8: Spectra display of incipient faults signals.
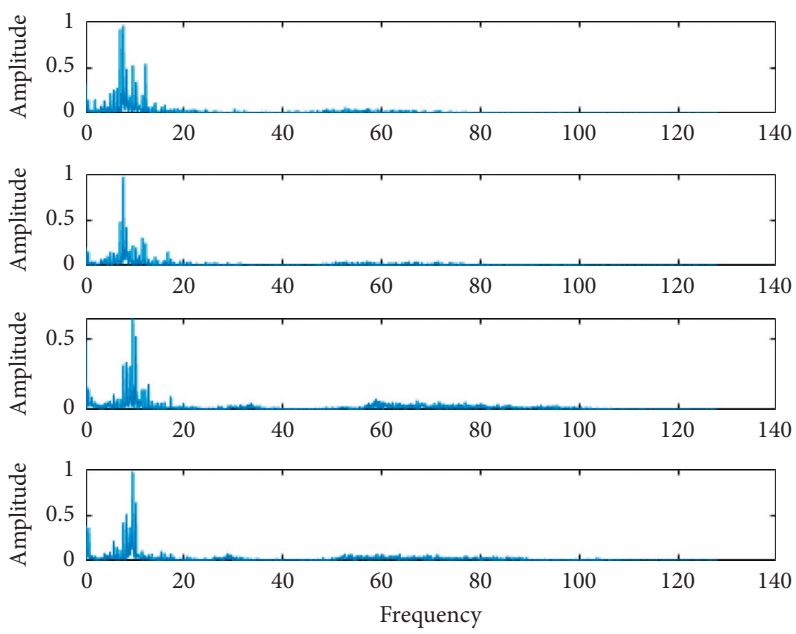

Figure 9: Spectra display of faults signals. 


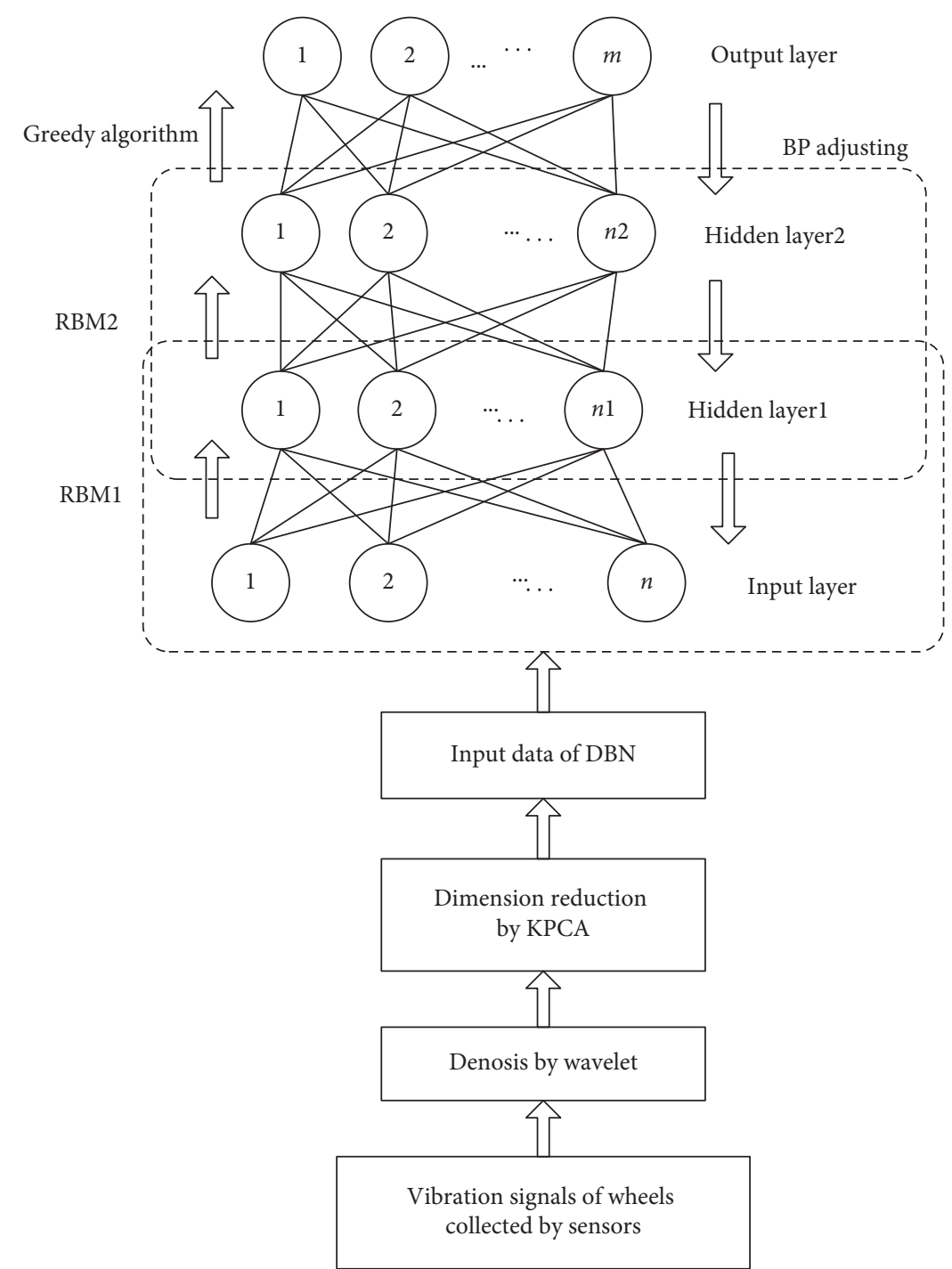

FIGURE 10: Whole network optimization.
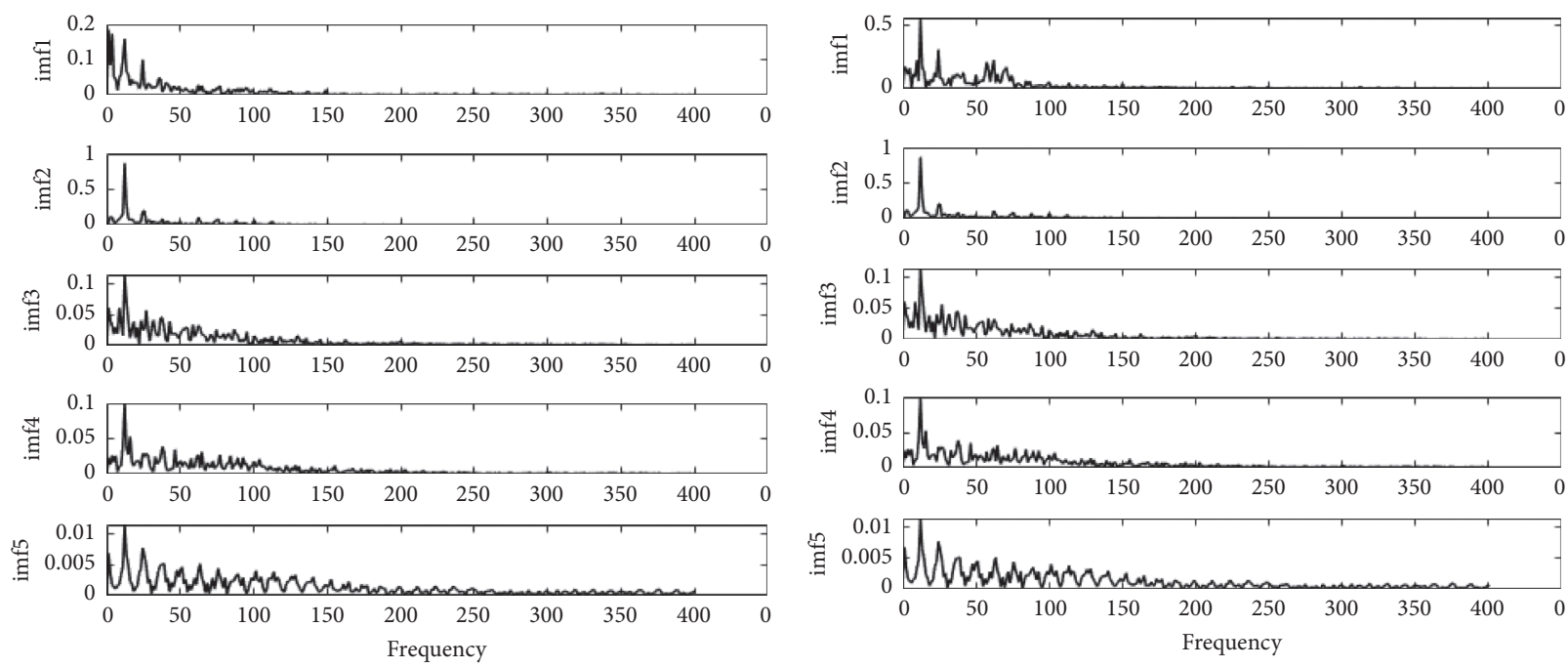

(a)

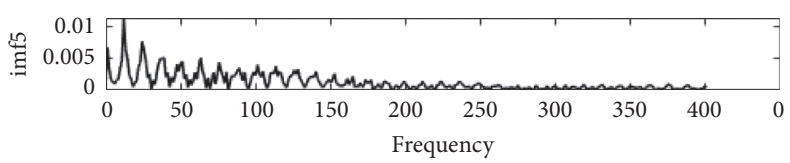

(b)

FIgURE 11: (a) Envelope diagram without denoise processing. (b) Envelope diagram of denoise processing by wavelet. 


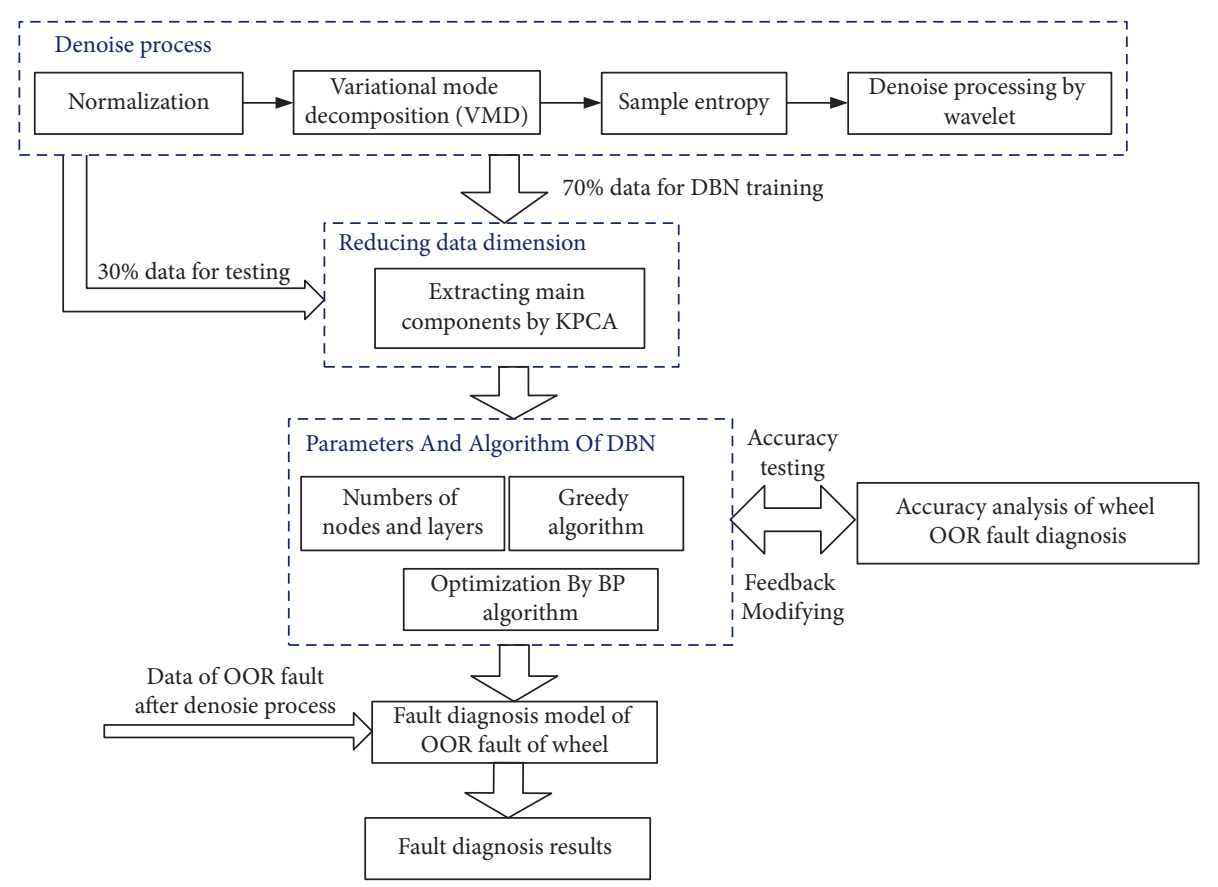

FIGURE 12: OOR faults diagnosis procedure of metro vehicle wheels.
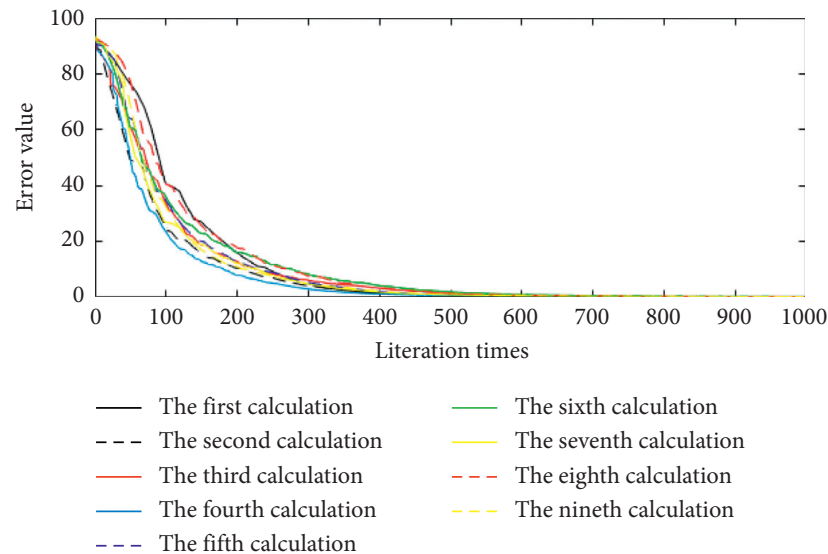

FIGURE 13: Errors declining with iterations for test 1.
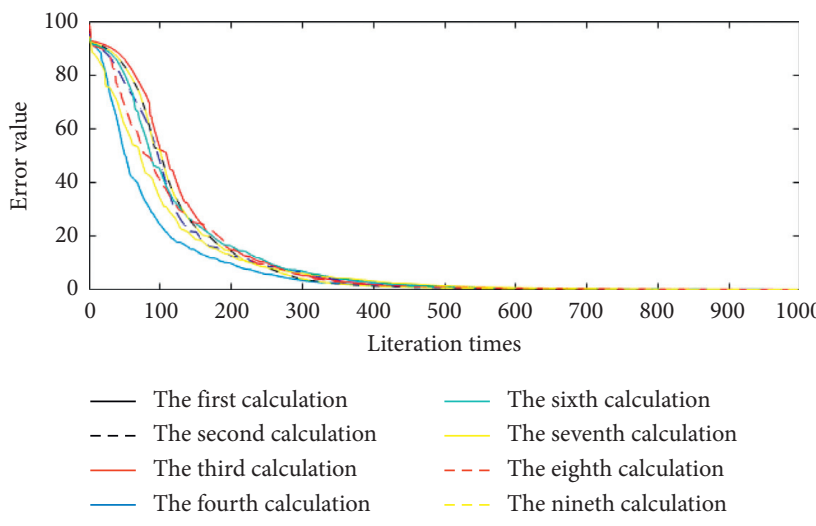

- _ - The fifth calculation

FIGURE 14: Errors declining with iterations for test 2. 

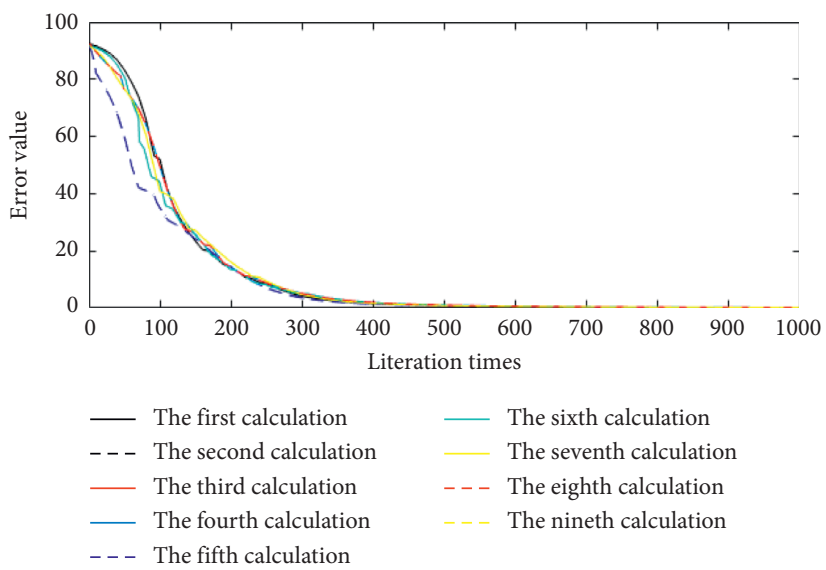

Figure 15: Errors declining with iterations for test 3.
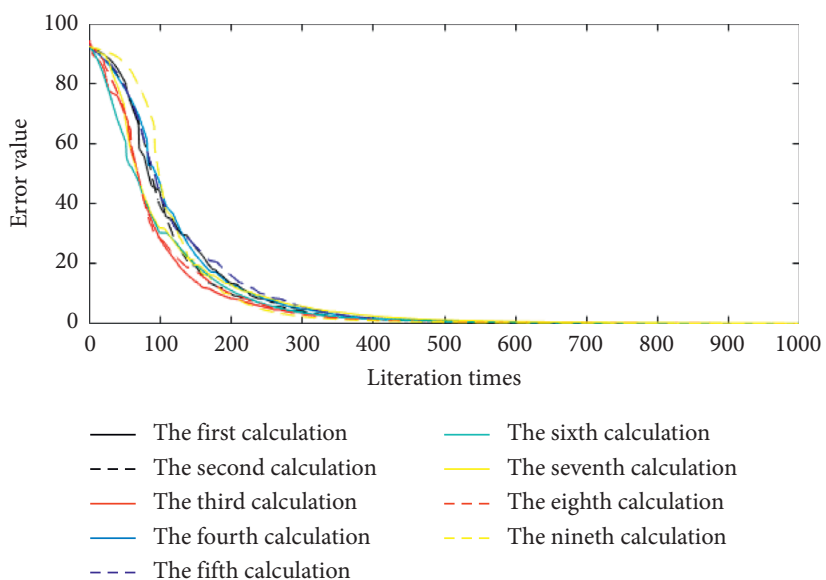

Figure 16: Errors declining with iterations for test 4.

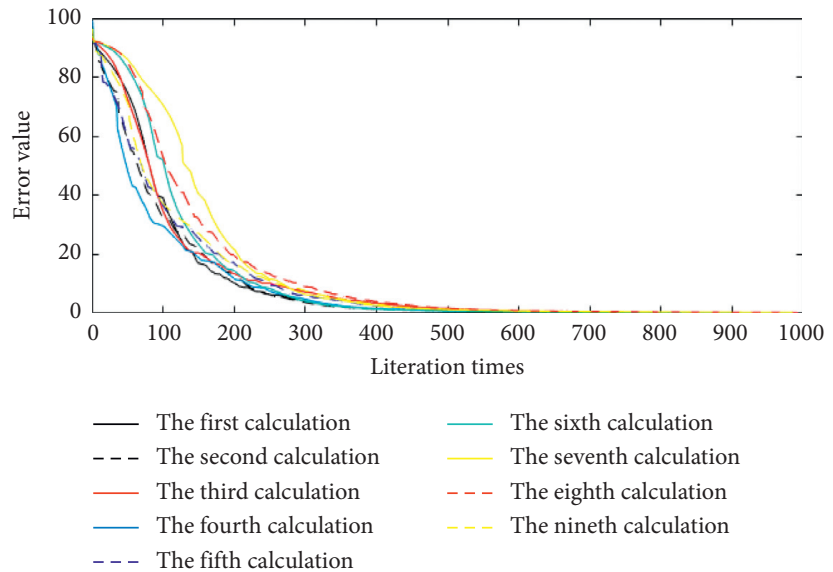

FIGURE 17: Errors declining with iterations for test 5. 


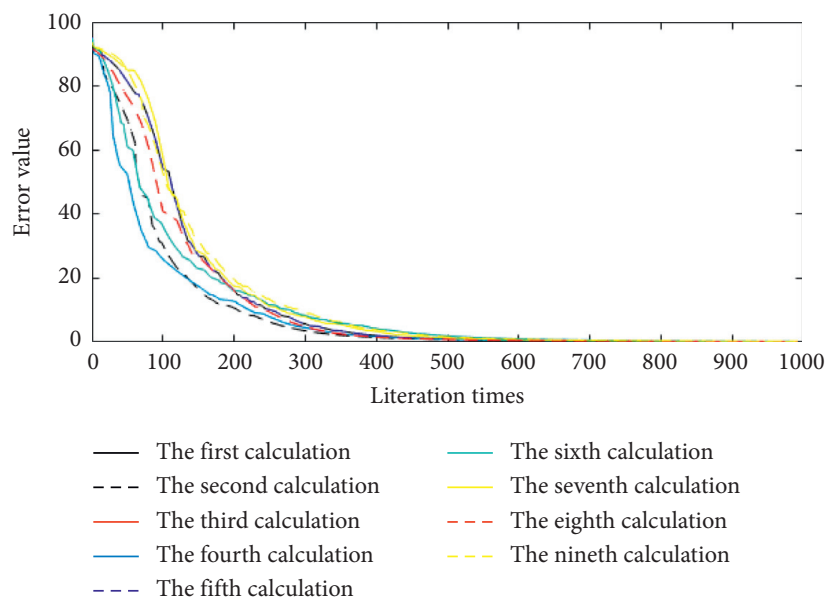

FIGURE 18: Errors declining with iterations for test 6.

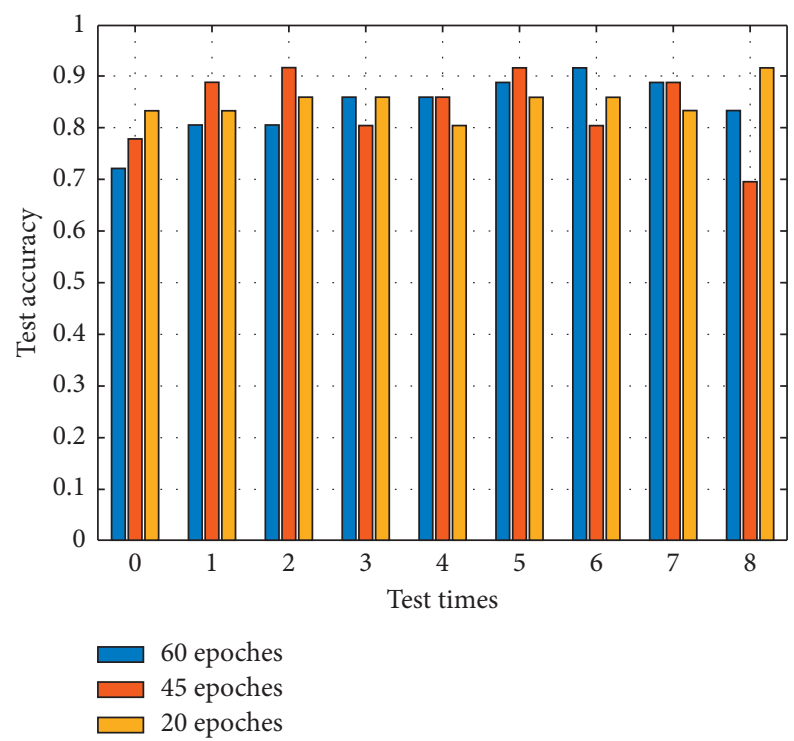

Figure 19: Calculation accuracy with different epochs of test 6 .

TAble 2: Parameters of diagnosis model and accuracies.

\begin{tabular}{|c|c|c|c|c|c|c|}
\hline Parameters & Test 1 & Test 2 & Test 3 & Test 4 & Test 5 & Test 6 \\
\hline Number of iterations & 1000 & 1000 & 1000 & 1000 & 1000 & 1000 \\
\hline Dimensions of KPCA & 40 & 20 & 25 & 22 & 25 & 22 \\
\hline Nodes of first layer & 50 & 30 & 20 & 30 & 30 & 30 \\
\hline Nodes of second layer & 30 & 20 & 20 & 20 & 20 & 30 \\
\hline Testing accuracy & 0.8057 & 0.8611 & 0.8889 & 0.9444 & 0.9722 & 0.9444 \\
\hline Learning rate & 0.001 & 0.001 & 0.001 & 0.001 & 0.001 & 0.001 \\
\hline Average accuracy of 9 times & 0.8426 & 0.8704 & 0.8481 & 0.8364 & 0.8858 & 0.9136 \\
\hline
\end{tabular}

TABle 3: Testing accuracies of different tests by nine times calculation.

\begin{tabular}{lcccccccccc}
\hline Test & 1st & 2nd & 3rd & 4th & 5th & 6th & 7th & 8th & 9th & Mean value \\
\hline Test 1 & 0.8056 & 0.7500 & 0.9167 & 0.7500 & 0.8056 & 0.9444 & 0.8889 & 0.8333 & 0.8888 \\
Test 2 & 0.8611 & 0.9167 & 0.8611 & 0.8333 & 0.9167 & 0.8611 & 0.9167 & 0.7778 & 0.8889 \\
Test 3 & 0.8889 & 0.9444 & 0.8056 & 0.8166 & 0.8056 & 0.9444 & 0.8056 & 0.8166 & 0.8056 \\
Test 4 & 0.9444 & 0.8611 & 0.8056 & 0.8889 & 0.8056 & 0.7778 & 0.8333 & 0.7778 & 0.8333 \\
Test 5 & 0.9722 & 0.8056 & 0.8056 & 0.9167 & 0.9167 & 0.8889 & 0.8333 & 0.9167 & 0.9167 \\
Test 6 & 0.9444 & 0.8333 & 0.9722 & 0.8611 & 0.8889 & 0.9444 & 0.9722 & 0.9444 & 0.8611 \\
\hline
\end{tabular}




\section{Conclusion}

We present a diagnosis method of out-of-round (OOR) faults of metro vehicle wheels that combines kernel principal component analysis (KPCA) and deep belief network (DBN), named KPCA-DBN diagnosis model, and an analysis to detect OOR faults of wheels even when signal-tonoise ratios (SNRs) are below 5. A KPCA-DBN diagnosis model is used to distinguish wheels with incipient and serious OOR faults [24].

Vibration signals collected by vibration sensors on running metro vehicles are used to train and test KPCA-DBN diagnosis model. VMD and wavelet methods are used to extract the main components and improve SNRs of vibration signals. Compare the sample entropy to judge the components with strong noises and adjust the parameters of the KPCA-DBN diagnosis model and try to get the optimal parameters by calculating test accuracy. Further, match six groups of parameters, including dimensions of KPCA, number of iterations, nodes of first layer, nodes of second layer, learning rate, and epochs. The mean testing accuracy is the best accuracy and is set at 0.9136 by the diagnosis model with the parameters of test 6 . Compared with other tests, there is a better generalization by test 6 . Analyze the differences of error decline going with nine iteration times in all tests. Epoch number is set at 22, which is better than 45 and 60, compared with mean test accuracy. When epoch number is 45 and 60 , there may be overlapping during training the diagnosis model.

It is found that the KPCA-DBN diagnosis method can enhance SNRs of vibration signals of metro vehicle wheels and detect incipient ORR faults during metro vehicle running, which allows accurate detection of such early-stage ORR faults of metro vehicle wheels.

The mean accuracy of diagnosis model proposed in this paper can be 0.9136 , and the diagnosis model presented in this paper is very significant to detect OOR faults online.

In conclusion, we presented a method to diagnose outof-round faults of train wheels, which can detect the faults on time for making reasonable maintenance scheme. A reasonable maintenance scheme will help reduce the cost of maintenance and stopping time of trains and improve stability and safety while trains are running.

This method may also be extended to diagnose OOR faults of high speed train, railway wagon, and other rail transit vehicles.

\section{Data Availability}

The data used to support the findings of this study are available from the corresponding author upon request.

\section{Conflicts of Interest}

The authors declare they have no conflicts of interest.

\section{Authors' Contributions}

The study was guided by Huang Haifeng and written by all authors.

\section{Acknowledgments}

This work was supported by the Science and Technology Plan Project of Sichuan Province (no. 19GJHZ0061). The authors express their sincere gratitude to Advances in Artificial Intelligence-IBERAMIA 2002 for the inspiration.

\section{References}

[1] Q. Lan, M. Dhanasekar, and Y. A. Handoko, "Wear damage of out-of-round wheels in rail wagons under braking," Engineering Failure Analysis, vol. 102, no. 8, pp. 170-186, 2019.

[2] N. Nencho, D. Emil, V. Vasil, and P. Petyo, "Sensor system of detecting defects in wheels of railway vehicles running at operational speed," in Proceedings of the ISSE: 34th International Spring Seminar on IEEE, pp. 577-825, Tratanska Lomnica, Slovakia, May 2011.

[3] O. Kılınç and J. Vágner, "Vibration based diagnosis of wheel defects of metro train sets using one period analysis on the wayside," Vibroengineering PROCEDIA, vol. 11, pp. 13-18, 2017.

[4] Z. An, Y. Zhang, M. Lu, and Q. Meng, "Reliability analysis of railway freight car wheelset based on Birnbaunm-Saunders fatigue life distribution," Vibroengineering PROCEDIA, vol. 35, pp. 88-93, 2020.

[5] X. Y. Ren and J. Y. Wu, "Construction of fault diagnosis model of metro wheel speed box system based on convolution neural network," in Proceedings Of ICAPC 2020: International Conference on Applied Physics and Computing, pp. 1-7, Ottawa, Canada, September 2020.

[6] E. M. Vinberg, M. Martin, A. H. Firdaus, and Y. Tang, A. Qazizadeh, Railway Applications of Condition Monitoring, pp. 1-49, KTH Royal Institute of Technology, Stockholm, Sweden, 2018.

[7] R. R. Song, W. H. Ma, and S. H. Luo, "Out-of-round wheels and the correlation problem of metro vehicles," in Proceedings of 1st International Conference On Transportation Engineering, pp. 1759-1764, Chengdu, China, July 2007.

[8] G. Tao, L. Wang, Z. Wen, and Q. Jin, "Measurement and assessment of out-of-round electric locomotive wheels," Proceedings of the Institution of Mechanical Engineers-Part F: Journal of Rail and Rapid Transit, vol. 232, no. 1, pp. 275-287, 2016.

[9] N. Fu, K. J. Qian, and Z. Y. Xing, "Urban rail train wheel fault diagnosis based on improved EEMD," in Proceedings of International Conference On Electrical And Information Technologies For Rail Transportation, Springer, Qingdao, China, October 2019.

[10] J. Zhang, C. L. Huang, and S. W. Zhou, "An approach to the characteristic spectrum analysis for train wheelset fault diagnosis," Key Engineering Materials, vol. 460-461, pp. 153158, 2011.

[11] G. Adam, "Ventilation diagnosis of angle grinder using thermal imaging," Sensors, vol. 21, p. 2853, 2021.

[12] O. AlShorman, F. Alkahatni, M. Masadeh et al., W. Glowacz, Sounds and acoustic emission-based early fault diagnosis of induction motor: a review study," Advances in Mechanical Engineering, vol. 13, no. 2, pp. 1-19, 2021.

[13] G. Wang, Y. Lv, T. Wang, X. Wang, and H. Cheng, "Mass laplacian discriminant analysis and its application in gear fault diagnosis," Shock and Vibration, vol. 2020, no. 6, 13 pages, Article ID 8826419, 2020. 
[14] B. Yang, Q. Li, L. Chen, C. Shen, and S. Natarajan, "Bearing fault diagnosis based on multilayer domain adaptation," Shock and Vibration, vol. 2020, Article ID 8873960, 11 pages, 2020.

[15] K. Zhu, P. Xun, W. Li, Z. Li, and R. Zhou, "Prediction of passenger flow in urban rail transit based on big data analysis and deep learning," IEEE Access, vol. 7, pp. 142272-142279, 2019.

[16] Y. Zou, Y. Zhang, and H. Mao, "Fault diagnosis on the bearing of traction motor in high-speed trains based on deep learning," Alexandria Engineering Journal, vol. 60, no. 1, pp. 1209-1219, 2021.

[17] Z. Yan, J. Han, L. Jing, Z. Tingliang, S. Junfeng, and L. Juan, "Safety prediction of rail transit system based on deep learning," in Proceedings of the 16th International Conference on Computer and Information Science (ICIS), pp. 851-856, Wuhan, China, June 2017.

[18] J. Yang, W. Bao, Y. Liu, X. Li, J. Wang, and Y. Niu, “A pairwise graph regularized constraint based on deep belief network for fault diagnosis," Digital Signal Processing, vol. 108, no. 3, pp. 102-119, 2021.

[19] A. Dedinec, S. Filiposka, A. Dedinec, and L. Kocarev, "Deep belief network based electricity load forecasting: an analysis of Macedonian case," Energy, vol. 115, no. 3, pp. 1688-1700, 2016.

[20] Z. Yemeni, J. Shu, X. Zhang, and L. Liu, “A DBN approach to predict the link in opportunistic networks," Recent Developments in Intelligent Computing, Communication and Devices, Springer, vol. vol. 752, pp. 575-587, Berlin, Germany, 2019.

[21] Y. Guo, W. Shuang, C. Gao, D. Shi, D. Zhang, and B. Hou, "Wishart RBM based DBN for polarimetric synthetic radar data classification," in Proceedings of 2015 IEEE International Geoscience and Remote Sensing Symposium, pp. 1841-1844, Milan, Italy, July 2015.

[22] J. Qiao, G. Pan, and H. Han, "A regularization-reinforced DBN for digital recognition," Natural Computing, vol. 18, no. 4, pp. 721-733, 2019.

[23] G. E. Hinton, S. Osindero, and Y. W. Teh, "A fast learning algorithm for deep belief nets," Neural Computation, vol. 18, no. 7, pp. 1527-1554, 2014.

[24] Z. Zhao, J. , Zhao, K. Song et al., "Joint DBN and fuzzy c-means unsupervised deep clustering for lung cancer patient stratification," Engineering Applications of Artificial Intelligence, vol. 91, no. 5, pp. 1-10, 2020. 